\title{
One man's trash is another man's treasure: um mapeamento sistemático sobre as características individuais na gamificação de ambientes virtuais de aprendizagem
}

\author{
Ana Carolina Tomé Klock ${ }^{1}$, Isabela Gasparini ${ }^{1}$, Avanilde Kemczinski ${ }^{1}$, \\ Marcelo da Silva Hounsell ${ }^{1}$, Seiji Isotani ${ }^{2}$ \\ ${ }^{1}$ PPGCA e PPGECMT, Departamento de Ciência da Computação \\ Universidade do Estado de Santa Catarina (UDESC) - Joinville - SC - Brasil \\ ${ }^{2}$ Instituto de Ciências Matemáticas e de Computação (ICMC) \\ Universidade de São Paulo (USP) - São Carlos - SP - Brasil \\ actklock@gmail.com, \{isabela.gasparini, marcelo.hounsell, \\ avanilde.kemczinski\}@udesc.br, sisotani@icmc.usp.br
}

\begin{abstract}
Gamification is a way to include game elements to a wide variety of applications and it is becoming popular in many contexts. The purpose of this paper is to verify from the literature if students with different characteristics react differently to the game elements of gamification through a systematic mapping. Three research questions were defined and, from them, a string used in seven academic search engines. 1355 papers were found and after application of inclusion and exclusion criteria, 7 papers were analyzed. From them, it was possible to identify that some characteristics (age, gender, motivations, players types) can influence the gamification of a virtual learning environment.
\end{abstract}

Resumo. A gamificação é uma forma de incluir elementos de jogos em diversas aplicações e está se tornando popular em vários contextos. $O$ objetivo desse artigo é verificar na literatura, através de um mapeamento sistemático, se os alunos com diferentes características reagem de forma diferente aos elementos de jogos da gamificação. Foram definidas três questões de pesquisas e, a partir delas, um argumento de busca foi utilizado em sete mecanismos de busca acadêmica. Foram encontrados 1355 artigos e, após os critérios de inclusão e exclusão, 7 artigos foram analisados. A partir deles, é possível identificar que algumas características (idade, gênero, motivações, tipos de jogadores) podem influenciar na gamificação de um ambiente virtual de aprendizagem.

\section{Introdução}

A área educacional amplia cada dia mais a utilização de recursos tecnológicos devido ao apoio que a tecnologia oferece, promovendo meios para que alunos e professores possam ter diferentes conexões interpessoais. Desta forma, o professor se transforma em um facilitador do processo de ensino-aprendizagem, e utiliza abordagens que considerem as características individuais dos alunos (como habilidades, necessidades e interesses) para auxiliar no aprendizado, flexibilizando o estudo e as estratégias didático-pedagógicas (Ismael; Al-Badi, 2014). Um exemplo são os sistemas hipermídia 
adaptativos (SHAs), que constroem um modelo com os objetivos, preferências e conhecimentos de cada usuário e utilizam esse modelo para adaptar a interação conforme as necessidades do usuário (Brusilovsky, 2012). Além dos SHAs, existem abordagens que podem engajar os alunos com o conteúdo, como a gamificação, que aplica elementos e design de jogos em contextos não-lúdicos a fim de estimular pessoas para atingir algum objetivo específico (Deterding et al., 2011).

Entretanto, alguns trabalhos, como o de Almaliki et al. (2014) e Codish et al. (2014), identificam que, conforme as características dos usuários, as técnicas de gamificação podem funcionar de maneira diferente. Dessa forma, o objetivo desse artigo é verificar quais características dos alunos mais influenciam na gamificação e sua relação com os elementos de jogos em ambientes virtuais de aprendizagem. Este trabalho realiza um mapeamento sistemático para identificar quais elementos de jogos são mais indicados de acordo com o perfil do aluno, visando um melhor aproveitamento do ambiente virtual de aprendizagem.

O artigo está estruturado da seguinte forma: na seção 2 é apresentado processo do mapeamento, definindo as questões de pesquisa, os mecanismos de busca utilizados, o argumento de busca e os critérios de inclusão e exclusão. Na seção 3 são feitas as análises e cada questão de pesquisa é discutida e respondida. A seção 4 apresenta as conclusões do mapeamento realizado.

\section{Processo do Mapeamento}

Um mapeamento sistemático é, de acordo com Petersen, Vakkalanka e Kuzniarz (2015), um processo que classifica e contabiliza as contribuições existentes em uma determinada área que possam dar uma visão geral da área de pesquisa.

Durante o planejamento de um mapeamento sistemático são definidas as questões de pesquisa, o processo de busca (que envolve a definição do argumento de busca com base nas questões de pesquisa e dos mecanismos de busca a serem utilizados), seguido pelo processo de seleção e qualificação dos artigos encontrados (através de critérios de inclusão e exclusão). Os artigos que passarem pela etapa de seleção e qualificação tem seus dados extraídos para responder as questões de pesquisa, sendo que esses dados são posteriormente analisados a fim de respondê-las.

\subsection{Definição das Questões de Pesquisa}

Para verificar se existem diferenças nas características dos usuários que tornem os elementos de gamificação mais ou menos indicados para os alunos, foram elaboradas as seguintes questões de pesquisa:

- $\quad \mathbf{Q P}_{1}$ : Quais características do usuário interferem no processo da gamificação em ambientes virtuais de aprendizagem?

- $\mathbf{Q P}_{2}$ : Quais elementos de jogos são mais indicados conforme as características do usuário?

- $\mathbf{Q P}_{3}$ : Quais técnicas de gamificação em ambientes educacionais podem ser adaptadas em função das características dos usuários? 


\subsection{Processo de Busca}

A partir das questões de pesquisa, o argumento de busca a ser utilizado nos mecanismos de busca foi definido. Para isso as questões de pesquisa foram detalhadas em três grandes categorias de palavras-chaves: relacionadas com gamificação (e.g., gamification, ludification, gamification), relacionadas com educação (e.g., education, learning, teaching, tutoring, students) e relacionadas com adaptabilidade $\mathrm{e}$ personalização (e.g., personality, culture, adaptability, style).

Entretanto, durante os testes nos mecanismos de busca, foi identificado que o leque de palavras-chaves relacionadas com as características do usuário é muito amplo para ser colocado no argumento de busca, e desta forma, optou-se por um argumento de busca mais genérico com apenas palavras-chaves relacionadas à gamificação. Assim, a busca foi realizada em artigos que contêm "gamification OR gamified OR gamify*” (sendo * equivalente ao caractere coringa) no título, resumo ou palavra-chave. Dessa forma, foi possível obter (após a filtragem) artigos que consideram alguma característica do usuário na gamificação na área educacional e também artigos potencialmente importantes de outras áreas de aplicação da gamificação.

Os mecanismos de busca acadêmicos utilizados foram ACM Digital Library, Science Direct, IEEE Xplore, Scopus, SpringerLink, Web of Science e Wiley Online Library. Para a seleção desses mecanismos, observamos outros mapeamentos sistemáticos existentes sobre gamificação, como o de Souza Borges et al. (2014) e o de Pedreira et al. (2015). As buscas foram realizadas em abril/2015 nos sete mecanismos descritos, com filtro de data de publicação (artigos a partir de 2010). Para a busca no SpringerLink foi necessário efetuar a filtragem por título, resumo e palavras-chave manualmente, pois a busca só pode ser realizada no texto completo. A quantidade de artigos retornada em cada mecanismo pode ser visualizada na Tabela 1.

Tabela 1. Quantidade de artigos retornados pelos Mecanismos de Busca Acadêmicos

\begin{tabular}{lr} 
Mecanismo de Busca & Quantidade \\
\hline ACM Digital Library & 242 \\
Science Direct & 48 \\
IEEE Xplore & 182 \\
Scopus & 602 \\
SpringerLink & 157 \\
Web of Science & 107 \\
Wiley Online Library & 17 \\
\hline Total & $\mathbf{1 3 5 5}$
\end{tabular}

Após a filtragem dos artigos retornados pelo mecanismo SpringerLink, a quantidade dos artigos diminuiu para 49 artigos no mecanismo e, no total, 1247 artigos foram identificados para passar pelo processo de seleção e qualificação.

\subsection{Processo de Seleção}

Com base nas questões de pesquisa, foram definidos critérios de inclusão e exclusão para que os artigos retornados pelas buscas fossem mais assertivos a fim de responder as questões. Como critérios de inclusão, além da data de publicação ser maior que 2009, foram considerados: 
- Artigos disponíveis para download;

- Artigos no idioma inglês;

- Artigos com trabalhos primários e completos (nesse caso, consideram-se completos artigos com quatro páginas ou mais).

Somente artigos que atenderam aos três critérios de inclusão foram avaliados conforme os critérios de exclusão:

- Artigos duplicados;

- Artigos não relacionados com a área educacional;

- Artigos que não identificam alguma característica do usuário.

Os artigos que atenderam algum dos critérios de exclusão foram removidos.

Tabela 2. Processo de aplicação dos critérios de inclusão e exclusão

\begin{tabular}{l|ccccccc|c}
\hline & $\begin{array}{c}\text { ACM } \\
\text { DL }\end{array}$ & $\begin{array}{c}\text { Science } \\
\text { Direct }\end{array}$ & $\begin{array}{c}\text { IEEE } \\
\text { Xplore }\end{array}$ & Scopus & $\begin{array}{c}\text { Springer } \\
\text { Link }\end{array}$ & $\begin{array}{c}\text { Web of } \\
\text { Scienc } \\
e\end{array}$ & $\begin{array}{c}\text { Wiley } \\
\text { OL }\end{array}$ & Total \\
\hline Após 2009 & 242 & 48 & 182 & 602 & 49 & 107 & 17 & 1247 \\
Disponíveis & 234 & 48 & 181 & 381 & 49 & 41 & 12 & 946 \\
Em inglês & 233 & 48 & 178 & 376 & 36 & 36 & 12 & 919 \\
Completos & 204 & 46 & 138 & 332 & 28 & 34 & 9 & 791 \\
\hline
\end{tabular}

Conforme Tabela 2, dos 1247 artigos retornados, apenas 791 artigos passaram pelos critérios de inclusão (artigos disponíveis, em inglês, primários e completos) e 486 desses artigos não estavam duplicados. Desses, foi possível verificar que 176 estão relacionados com a área educacional, mas apenas 19 citavam alguma característica do aluno no decorrer do artigo. Dos 19 artigos resultante, somente 7 artigos utilizavam efetivamente a característica citada para avaliar o processo de gamificação na educação.

\subsection{Processo de Extração dos Dados}

Dos sete artigos resultantes, foram extraídos dados para responder as questões de pesquisa e para identificar como as características dos alunos foram consideradas durante o estudo, em quais disciplinas foi utilizada a gamificação, quais elementos de jogos foram aplicados e qual a origem dos artigos.

Assim, foram analisados os seguintes dados para cada artigo: ano de publicação, país da filiação dos autores, base onde foi encontrado, onde foi publicado e o tipo desse veículo (periódico ou evento), objetivo do artigo, a disciplina onde foi aplicada a gamificação, os elementos de jogos utilizados, as características e quantidade dos alunos, as características avaliadas no artigo, o processo de avaliação, os resultados obtidos e as conclusões dos autores.

Por ano de publicação, os artigos foram publicados entre 2013 e 2015, sendo dois deles publicados em 2013, quatro em 2014 e um em 2015. Para verificar onde esse tema está sendo pesquisado, também foi identificado quais os países dos autores dos 
artigos. Dos sete artigos, dois deles foram escritos nos Estados Unidos, um em Portugal, um na Finlândia, um na Irlanda do Norte, um na África do Sul e um na China.

Por base e veículo de publicação, três artigos disponíveis no Science Direct foram publicados em periódicos (dois no periódico "Computers \& Education" e um no periódico "Procedia - Social and Behavioral Sciences") e quatro artigos foram publicados em eventos, sendo dois deles provenientes do ACM Digital Library (um no evento "First ACM SIGCHI annual symposium on Computer-Human Interaction in Play" e outro no evento "Southern African Institute for Computer Scientist and Information Technologists Annual Conference") e os outros dois do IEEE Xplore (um no evento "International Conference on Teaching and Learning in Computing and Engineering" e outro no evento "International Conference on Interactive Technologies and Games”), conforme apresentado no Gráfico 1.

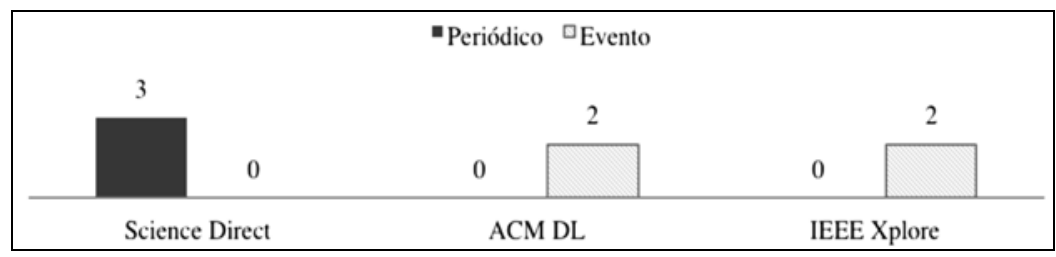

Gráfico 1. Artigos por veículo de publicação e base de origem

Entre os artigos selecionados, a maioria utiliza o tipo de jogador como uma característica para análise do funcionamento da gamificação em ambientes educacionais. Outros aspectos encontrados foram o sexo (gênero), a idade e a motivação. A Tabela 3 apresenta a quantidade de artigos por característica do aluno.

Tabela 3. Características dos alunos citadas em cada artigo

\begin{tabular}{l|c|c|c|c}
\hline Autores & Tipo de jogador & Sexo & Idade & Motivação \\
\hline Atalli et al. (2015) & & & $\mathrm{X}$ & \\
\hline Barata et al. (2014) & $\mathrm{X}$ & & & \\
\hline Christy et al. (2014) & & $\mathrm{X}$ & & \\
\hline Hakulinen et al. (2014) & & & & $\mathrm{X}$ \\
\hline Herbert et al. (2014) & $\mathrm{X}$ & & & \\
\hline O'Donovan et al. (2013) & $\mathrm{X}$ & & & \\
\hline Su et al. (2013) & & $\mathrm{X}$ & & $\mathbf{1}$ \\
\hline Total & $\mathbf{3}$ & $\mathbf{2}$ & $\mathbf{1}$ & $\mathbf{1}$ \\
\hline
\end{tabular}

Sobre as áreas de atuação do ambiente, ou seja, as disciplinas trabalhadas nos ambientes virtuais de aprendizagem, foi constatado que a maioria aplica a gamificação em disciplinas relacionadas com Computação (algoritmos, estrutura de dados, desenvolvimento web e desenvolvimento de jogos), mas também foi aplicada gamificação em disciplinas de Matemática e de Biologia, conforme Gráfico 2.

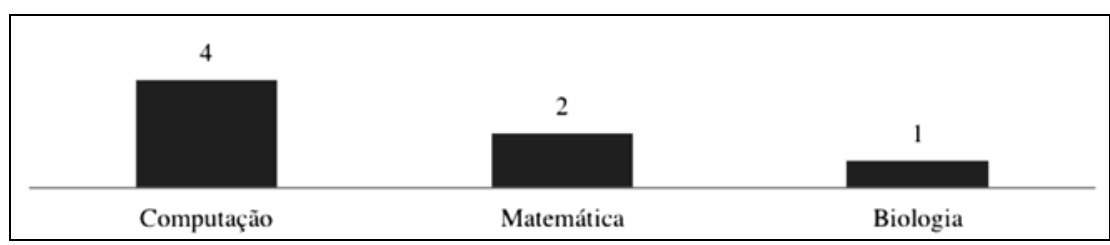

Gráfico 2. Artigos por disciplinas de aplicação 
Em relação aos elementos de jogos utilizados na gamificação dos ambientes educacionais, um dos artigos que analisava o sexo como característica não identificou quais elementos de jogos foram utilizados. Nos demais artigos, os elementos utilizados foram: pontos, medalhas e rankings (presentes em 4 artigos), níveis e feedback (presentes em 3 artigos), personalização e conquistas (presentes em 2 artigos) e narrativas, desafios e tentativas (presentes em 1 artigo), conforme Tabela 4.

Tabela 4. Elementos de jogos citados em cada artigo

\begin{tabular}{|c|c|c|c|c|c|c|c|}
\hline Elemento de Jogo & 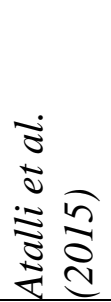 & 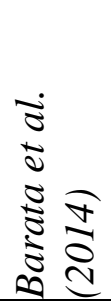 & 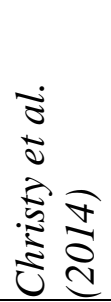 & 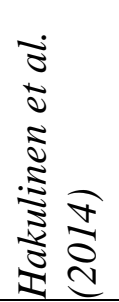 & 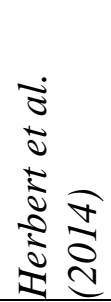 & 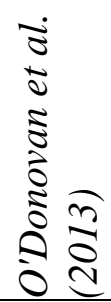 & Total \\
\hline Pontos & $\mathrm{X}$ & $\mathrm{X}$ & & & $\mathrm{X}$ & $\mathrm{X}$ & 4 \\
\hline Medalhas & & $\mathrm{X}$ & & $X$ & $\mathrm{X}$ & $\mathrm{X}$ & 4 \\
\hline Rankings & & $\mathrm{X}$ & $X$ & & $X$ & $\mathrm{X}$ & 4 \\
\hline Níveis & & $X$ & & & $\mathrm{X}$ & $X$ & 3 \\
\hline Feedback & & $\mathrm{X}$ & & & $\mathrm{X}$ & $\mathrm{X}$ & 3 \\
\hline Customização & & $\mathrm{X}$ & & & $\mathrm{X}$ & & 2 \\
\hline Conquistas & & $\mathrm{X}$ & & & $\mathrm{X}$ & & 2 \\
\hline Narrativa & & & & & & $X$ & 1 \\
\hline Desafios & & $\mathrm{X}$ & & & & & 1 \\
\hline Tentativas & & & & & & $X$ & 1 \\
\hline
\end{tabular}

Os sistemas de pontos são abertos, diretos e motivacionais, permitindo a utilização de vários tipos diferentes de pontuação, de acordo com o objetivo proposto. Os níveis indicam o progresso do usuário dentro do sistema. Reforço e feedback são recursos utilizados para prover dados importantes ao usuário, informando sua localização no ambiente e os resultados das ações realizadas por ele dentro do sistema. A customização oportuniza que usuários personalizem suas configurações no sistema. As conquistas são uma versão mais robusta de pontos e representam alguma realização/conquista do usuário no sistema. Elas são representadas pelas medalhas. As narrativas fazem com que o usuário apresente um comportamento esperado em um contexto. Os desafios são os elementos que orientam os usuários sobre as atividades que devem ser realizadas dentro de um sistema. Finalmente, as tentativas auxiliam o usuário/aluno, dando a oportunidade de que ele possa realizar uma tarefa novamente.

Unificando as características analisadas pelos autores (Tabela 3) e os elementos de jogos utilizados com base nessas características (Tabela 4), pode-se visualizar que trabalhos baseados no tipo de jogador utilizaram mais elementos de jogos durante 0 processo de gamificação do que os trabalhos que consideravam a idade, o sexo e as motivações dos alunos, conforme Gráfico 3. 
CBIE-LACLO 2015

Anais do XXVI Simpósio Brasileiro de Informática na Educação (SBIE 2015)

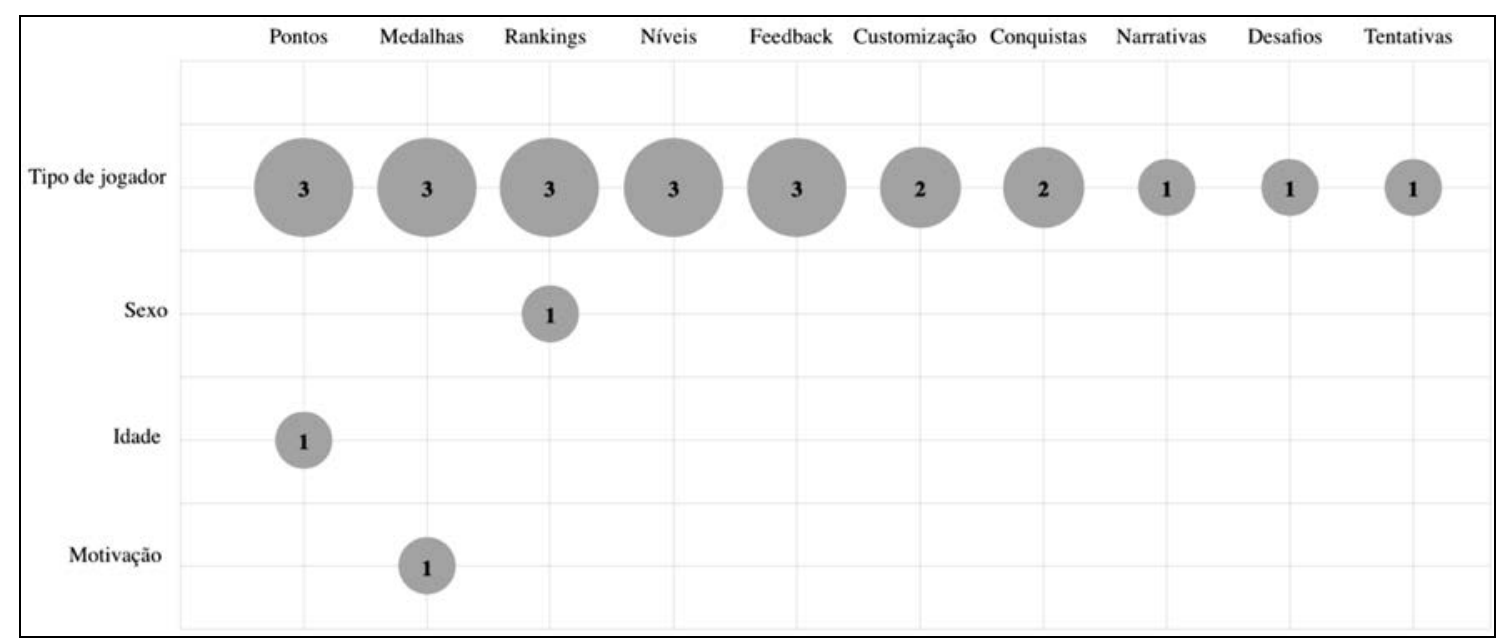

Gráfico 3. Elementos de jogos utilizados conforme características dos alunos

Os trabalhos que analisam características como sexo (Christy et al., 2014), idade (Attali et al., 2015) e motivações (Hakulinen et al., 2014) utilizam apenas um elemento de jogo para gamificar o ambiente virtual de aprendizagem. Apesar disso, o Gráfico 3 apenas apresenta quais elementos de jogos foram utilizados para cada característica analisada, sem entanto analisar seu significado (se as técnicas são positivas ou não para a experiência que o aluno tem no sistema).

\section{Discussão}

Através dos sete artigos encontrados na literatura, foi possível identificar que existem características dos alunos que podem interferir no processo de gamificação de ambientes educacionais. Uma das características que pode interferir é a idade, de acordo com Attali et al. (2015), pois através do seu estudo notou-se que adolescentes estão mais engajados do que adultos durante a utilização do ambiente educacional gamificado com pontos.

Outra característica que pode interferir no processo de gamificação é o sexo, segundo Christy et al. (2014) e Su et al. (2013). O trabalho de Christy et al. (2014) utiliza rankings para avaliar o engajamento das alunas de uma turma de matemática, colocando uma parte das alunas para visualizar o ranking com predominância de nomes masculinos, enquanto outra parte das alunas visualizava o ranking com a predominância de nomes femininos. Nesse experimento, as alunas que visualizaram o ranking com predominância de nomes masculinos obtiveram notas maiores nos testes de conhecimento do que as alunas que visualizaram o ranking com predominância de nomes femininos (Christy et al., 2014). Em contrapartida, as alunas que visualizaram o ranking com predominância de nomes femininos tiveram uma melhor identificação acadêmica (que envolvem preferências acadêmicas, como gostar ou não de ir para aula), mensurada através de um questionário. O trabalho de Su et al. (2013) realiza um experimento sobre conhecimento de insetos, e destaca que a gamificação é mais motivadora para alunos do sexo masculino do que para alunas do sexo feminino, mas não explicitam quais foram os elementos de jogos utilizados em seu ambiente educacional. 
O trabalho de Hakulinen et al. (2014) explora as motivações dos alunos através de uma conceituação da psicologia chamada "Conquista orientada à meta", que caracteriza as preferências dos alunos conforme seus objetivos e resultados. As metas podem ser orientadas ao domínio (dominar uma tarefa) ou ao desempenho (mostrar competência em relação aos demais). Segundo Hakulinen et al. (2014), essas metas podem ainda ser subdivididas pelas preferências entre o domínio intrínseco (aprender novos conhecimentos), o domínio extrínseco (ter sucesso na escola), a prova de desempenho (ser melhor do que os demais estudantes) e a fuga do desempenho (evitar situações onde podem ocorrer erros e enganos). Segundo Hakulinen et al. (2014) as metas não são mutuamente exclusivas, mas sim uma composição de metas em diferentes intensidades. Os autores expõem que a motivação do aluno pode interferir no processo de gamificação, pois os alunos motivados pelo domínio intrínseco, pelo domínio extrínseco e pela prova de desempenho tendem a ser mais engajados por medalhas do que alunos motivados pela fuga do desempenho.

O tipo de jogador também pode influenciar no processo de gamificação, conforme os trabalhos de Herbert et al. (2014), Barata et al. (2014) e O’Donavan et al. (2013). O trabalho de Hebert et al. (2014) cria e testa em sala de aula um questionário que auxilia na identificação dos tipos de jogadores conforme a tipologia proposta por Marczewski (2013), uma tipologia específica para aplicação em gamificação, que se baseia nas motivações dos jogadores (podendo ser relacionamento, autonomia, superioridade, propósito, recompensas e mudanças) e auxilia na escolha dos elementos de jogos a partir dessas motivações. O trabalho de Barata et al. (2014) divide os alunos em quatro categorias: 1) Achievers, estudantes que fazem de tudo para ganhar pontos e são beneficiados pela maioria dos elementos de jogos; 2) Regular Students, motivados por desafios, missões e conquistas; 3) Halfhearted Students, estudantes motivados apenas por desafios e missões e; 4) Underachievers, que não se motivam com elementos de jogos e fazem apenas o suficiente para passar na disciplina. Já o trabalho de O’Donavan et al. (2013) utiliza a tipologia BrainHex de Nacke et al. (2011) e afirma que os elementos mais motivadores para alunos com perfis de jogador que gostam de estar no controle (sem medo, ao contrário do tipo Survivor), que não gostam de jogar com outras pessoas (sem misericórdia, ao contrário do tipo Socialiser) e que não gostam de estar sob pressão (sem pressão, ao contrário do tipo Daredevil) são rankings, pontos, barras de progresso e medalhas (nessa ordem).

É possível verificar que as características do usuário estudadas nos artigos analisados são tipos de jogadores, sexo, idade, motivação. Essas características podem interferir no processo da gamificação em ambientes virtuais de aprendizagem $\left(\mathbf{Q P}_{\mathbf{1}}\right)$ e que, de acordo com essas características, existem elementos de jogos mais indicados $\left(\mathbf{Q P}_{2}\right)$. Apesar de nenhum trabalho discutir sobre a adaptabilidade do sistema na gamificação, acredita-se ser possível realizar uma adaptação dos elementos de jogos com base nas características dos alunos, dando enfoque nos elementos de jogos que mais motivam os alunos com as mesmas características $\left(\mathbf{Q P}_{3}\right)$, visto os resultados dos trabalhos. Entretanto, percebe-se a dificuldade em levantar as diferentes características dos alunos e analisar como essas estão relacionadas a cada elemento de gamificação. Automatizar esse processo tornando-o transparente para o usuário é uma tarefa árdua, porém, que pode aumentar o engajamento e motivação dos alunos nos ambientes virtuais de aprendizagem por promover mais personalização no ambiente. 


\section{Conclusão}

Ao decorrer deste mapeamento sistemático, foi verificado que alunos com diferentes características podem reagir de forma diferente aos elementos da gamificação. $\mathrm{O}$ processo de busca resultou em 1355 artigos, dos quais 178 estavam relacionados à educação e 19 citavam alguma característica dos alunos. Entretanto, apenas sete desses artigos fizeram uso efetivo da característica citada na verificação do processo de gamificação.

Nesses sete artigos, foi possível verificar que a idade é um fator que impacta no processo de gamificação, sendo que adolescentes são mais engajados com a utilização de pontos do que adultos. Outro fator que impacta é o sexo, pois estudantes do sexo masculino tendem a ser mais motivados do que estudantes do sexo feminino. A motivação do aluno também pode interferir no processo, pois alunos motivados pela a fuga do desempenho (que evitam o estresse) não se sentiram tão engajados com o uso de medalhas, ao contrário de estudantes que são motivados pelo domínio intrínseco, pelo domínio extrínseco e pela prova de desempenho. O tipo de jogador de cada estudante também influencia na gamificação, pois cada jogador interage de maneira distinta no ambiente e pode ser mais ou menos motivado com os elementos disponíveis no ambiente. Considerando todas as características dos alunos, os elementos de jogos mais estudados foram pontos, medalhas e rankings, sendo que cada elemento tem suas peculiaridades em relação as características individuais dos alunos: o que não serve para motivar um aluno ("trash") pode ser muito importante para a motivação de outro ("treasure"), i.e. "one man's trash is another man's treasure". Assim, esse artigo conseguiu identificar artigos na literatura que consideram as características dos alunos na aplicação da gamificação e quais elementos de jogos são mais indicados. Ainda, foi possível visualizar que, apesar da área ser recente, o tema tem sido estudado em diferentes países e é discutido por eventos científicos e periódicos.

Nenhum dos artigos levantados faz alguma adaptação da gamificação no ambiente virtual de aprendizagem. Acreditamos que este é um trabalho árduo mas possível, através do conhecimento das características dos alunos e dos elementos de jogos mais recomendados para essas características. Desta forma, verifica-se uma oportunidade de pesquisa que pode unir a área de gamificação com os sistemas hipermídia adaptativos, oportunidade essa que será investigada em trabalhos futuros.

\section{Referências}

Almaliki, M., Jiang, N., Ali, R. and Dalpiaz, F. (2014). Gamified Culture-aware Feedback Acquisition. In Proceedings of the 2014 IEEE/ACM 7th International Conference on Utility and Cloud Computing. . IEEE Computer Society.

Attali, Y., Arieli-Attali, M. (2014). Gamification in Assessment: Do Points Affect Test Performance?. In Computers \& Education, v. 83, pp. 57-63, Elsevier.

Barata, G., Gama, S., Jorge, J. A., Gonçalves, D. J. (2014). Relating gaming habits with student performance in a gamified learning experience. In Proceedings of the first ACM SIGCHI annual symposium on Computer-Human Interaction in Play, pp. 1725, ACM. 
Brusilovsky, P (2012). Adaptive hypermedia for education and training. Adaptive technologies for training and education, pp. 46-68, Cambridge University Press.

Christy, K. R., Fox, J. (2014). Leaderboards in a virtual classroom: A test of stereotype threat and social comparison explanations for women's math performance. In Computers \& Education, v. 78, pp. 66-77, Elsevier.

Codish, D. and Ravid, G. (2014). Personality based gamification-Educational gamification for extroverts and introverts. In Proc. 9 th Chais Conf. for the Study of Innovation and Learning Technologies: Learning in the Technological Era.

Deterding, S., Dixon, D., Nacke, L.E., Khaled, R. (2011). Gamification: Toward a definition. In: Conference on Human Factors in Computing Systems, pp. 9-15, ACM.

Hakulinen, L., Auvinen, T. (2014). The effect of gamification on students with different achievement goal orientations. In 2014 International Conference on Teaching and Learning in Computing and Engineering (LaTiCE), pp. 9-16, IEEE.

Herbert, B., Charles, D., Moore, A., Charles, T. (2014). An Investigation of Gamification Typologies for Enhancing Learner Motivation. In 2014 International Conference on Interactive Technologies and Games (iTAG), pp. 71-78, IEEE.

Ismael, S. M, Al-Badi, A. H. (2014). Technology for Enhancing the Learning and Teaching. In International Journal of Social, Education, Economics and Management Engineering, v. 8, no. 8, pp. 2439-2447, World Academy of Science, Engineering and Technology.

Marczewski, A. (2013). Gamification User Types. [Online]. Disponível em: http://marczewski.me.uk/user-types/. Último acesso: 25 mai. 2015.

Nacke, L. E., Bateman, C., Mandryk, R. L. (2011). BrainHex: preliminary results from a neurobiological gamer typology survey. In Entertainment Computing - ICEC 2011, pp. 288-293, Springer Berlin Heidelberg.

O'Donovan, S., Gain, J., Marais, P. (2013). A case study in the gamification of a university-level games development course. In Proceedings of the South African Institute for Computer Scientists and Information Technologists Conference, pp. 242-251, ACM.

Pedreira, O., García, F., Brisaboa, N., Piattini, M. (2015). Gamification in software engineering - A systematic mapping. In Information and Software Technology, v. 57, pp. 157-168, Elsevier.

Petersen, K., Vakkalanka, S., Kuzniarz, L. (2015). Guidelines for conducting systematic mapping studies in software engineering: An update. In Information and Software Technology, v. 64, pp. 1-18, Elsevier.

Sousa Borges, S. de, Durelli, V. H., Reis, H. M., Isotani, S. (2014). A systematic mapping on gamification applied to education. In Proceedings of the 29th Annual ACM Symposium on Applied Computing, pp. 216-222, ACM.

Su, C. H., Cheng, C. H. (2013). A Mobile Game-based Insect Learning System for improving the learning achievements. In Procedia - Social and Behavioral Sciences, v. 103, pp. 42-50, Elsevier. 